\title{
On the Distribution of the First Exit Time and Overshoot in a Two-Sided Boundary Crossing Problem
}

\author{
V. I. Lotov ${ }^{1 *}$ and A. S. Tarasenko ${ }^{2 * *}$ \\ ${ }^{1}$ Sobolev Institute of Mathematics, Novosibirsk State University, Novosibirsk, 630090 Russia \\ ${ }^{2}$ Novosibirsk State University, Novosibirsk, 630090 Russia \\ Received December 24, 2011
}

\begin{abstract}
We consider a random walk generated by a sequence of independent identically distributed random variables. We assume that the distribution function of a jump of the random walk equals an exponential polynomial on the negative half-axis. For double transforms of the joint distribution of the first exit time from an interval and overshoot, we obtain explicit expressions depending on finitely many parameters that, in turn, we can derive from the system of linear equations. The principal difference of the present article from similar results in this direction is the rejection of using factorization components and projection operators connected with them.
\end{abstract}

DOI: $10.3103 / \mathrm{S} 105513441302003 \mathrm{X}$

Keywords: random walk, two-sided boundary crossing problem, moment generating functions.

\section{INTRODUCTION}

We consider a sequence of independent identically distributed random variables $\left\{X_{n}, n \geq 1\right\}$. Let $S_{0}=0, S_{n}=X_{1}+\cdots+X_{n}, n \geq 1$. Given arbitrary $a>0$ and $b>0$, we introduce the first exit time from the interval $[-a, b)$ for the random walk $\left\{S_{n}\right\}$ as is follows:

$$
N=\min \left\{n \geq 1: S_{n} \notin[-a, b)\right\} .
$$

In the present paper, we study the joint distributions

$$
\mathbb{P}\left(S_{n} \in d x, N>n\right) \text { and } \mathbb{P}\left(S_{N} \in d x, N=n\right) .
$$

The list of the papers connected somehow with the study of the moment of the first exit of a random walk from an interval is so numerous that it is impossible to expound the history of the problem in the framework of this article. We only remark that the most part of the studies in this direction relates to various approximations of the distributions under consideration, including proving limit theorems and obtaining asymptotic expansions in different schemes of the asymptotic analysis (for instance, if $a+b \rightarrow \infty$ or in a triangle array scheme with decreasing jump sizes). We shift our accent to asymptotic results since finding explicit formulas for the joint distribution of the pair $\left(N, S_{N}\right)$ is available only for some classes of jump distributions of rather particular forms. More precisely, the probabilities $\mathbb{P}\left(S_{N} \geq b\right)$ and $\mathbb{P}(N=n)$ are well known in the simplest version of the ruin problem when $\mathbb{P}\left(X_{1}=1\right)+\mathbb{P}\left(X_{1}=\right.$ $-1)=1$ [5], the explicit expressions are found for $\mathbb{P}\left(N=n, S_{N}=k\right)$ in the case when $X_{1}$ has the twosided geometric distribution (see [4]). In the last case and in the situation when jumps of a random walk have an exponential density on each half-line, the explicit expressions are known for the moment generating functions $\sum z^{n} \mathbb{P}\left(N=n, S_{N} \in A\right)$ and, as a corollary, for the probabilities $\mathbb{P}\left(S_{N} \in A\right)$ (see

\footnotetext{
${ }^{*}$ E-mail: lotov@math.nsc.ru

**E-mail: dkanus@gmail.com
} 
$[2,3])$. It is possible to obtain much more results in finding double transforms of the sought distributions in the two-sided boundary crossing problem. Introduce the following notation:

$$
\begin{array}{ll}
Q_{0}(z, \lambda)=1+\sum_{n=1}^{\infty} z^{n} \int_{-a}^{b} e^{\lambda x} \mathbb{P}\left(S_{n} \in d x, N>n\right), & |z|<1, \operatorname{Re} \lambda=0, \\
Q_{1}(z, \lambda)=\sum_{n=1}^{\infty} z^{n} \int_{-\infty}^{-a} e^{\lambda x} \mathbb{P}\left(S_{N} \in d x, N=n\right), & |z| \leq 1, \operatorname{Re} \lambda \geq 0, \\
Q_{2}(z, \lambda)=\sum_{n=1}^{\infty} z^{n} \int_{b}^{\infty} e^{\lambda x} \mathbb{P}\left(S_{N} \in d x, N=n\right), & |z| \leq 1, \operatorname{Re} \lambda \leq 0 .
\end{array}
$$

It is well known [7] that these functions are connected by the relation

$$
r(z, \lambda) Q_{0}(z, \lambda)=1-Q_{1}(z, \lambda)-Q_{2}(z, \lambda),
$$

where

$$
r(z, \lambda)=1-z \varphi(\lambda), \quad \varphi(\lambda)=\mathbb{E} e^{\lambda X_{1}}, \quad \operatorname{Re} \lambda=0, \quad|z|<1 .
$$

The application of a Wiener-Hopf type method allows us to solve this equation. The method is based on the factorization (which is often attributed to the names of N. Wiener and E. Hopf)

$$
r(z, \lambda)=R_{+}(z, \lambda) R_{-}(z, \lambda), \quad|z|<1, \operatorname{Re} \lambda=0,
$$

where we can put

$$
\begin{aligned}
& R_{-}(z, \lambda)=\exp \left\{-\sum_{n=1}^{\infty} \frac{z^{n}}{n} \mathbb{E}\left(\exp \left\{\lambda S_{n}\right\} ; S_{n}<0\right)\right\}, \\
& R_{+}(z, \lambda)=\exp \left\{-\sum_{n=1}^{\infty} \frac{z^{n}}{n} \mathbb{E}\left(\exp \left\{\lambda S_{n}\right\} ; S_{n} \geq 0\right)\right\} .
\end{aligned}
$$

The functions $Q_{i}$ will be expressed in terms of the operators $\mathcal{A}$ and $\mathcal{B}$ that are defined by using the factorization components $R_{ \pm}(z, \lambda)$. For every function $g$ of the form

$$
g(\lambda)=\int_{-\infty}^{\infty} e^{\lambda y} d G(y), \quad \operatorname{Re} \lambda=0, \quad \int_{-\infty}^{\infty}|d G(y)|<\infty
$$

for $|z|<1$ and $\operatorname{Re} \lambda=0$, we put

$$
\begin{aligned}
& \mathcal{A} g(z, \lambda)=R_{-}(z, \lambda)\left[R_{-}^{-1}(z, \lambda) g(\lambda)\right]^{(-\infty,-a)}, \\
& \mathcal{B} g(z, \lambda)=R_{+}(z, \lambda)\left[R_{+}^{-1}(z, \lambda) g(\lambda)\right]^{[b, \infty)},
\end{aligned}
$$

where we use the conventional notation

$$
\left[\int_{-\infty}^{\infty} e^{\lambda y} d G(y)\right]^{D}=\int_{D} e^{\lambda y} d G(y) .
$$

Then $($ see $[2,3,7])$

$$
\begin{aligned}
& Q_{1}(z, \lambda)=\mathcal{A} e(z, \lambda)-\mathcal{A B} e(z, \lambda)+\mathcal{A B} Q_{1}(z, \lambda) \\
& Q_{2}(z, \lambda)=\mathcal{B} e(z, \lambda)-\mathcal{B} \mathcal{A} e(z, \lambda)+\mathcal{B} \mathcal{A} Q_{2}(z, \lambda)
\end{aligned}
$$

where $e(\lambda) \equiv 1$.

Formulas (2) and (3) enable us to find the asymptotic representations for the functions $Q_{i}$ under the Cramér conditions as $a \rightarrow \infty$ and $b \rightarrow \infty[2,3]$ and obtain the representations of these functions in the form of series containing powers of the projection operators $\mathcal{A}$ and $\mathcal{B}$. However, these formulas are of little use to be explicit expressions for double transforms because the factorization components and projection operators connected with them have rather complicated structures. 
The above reveals the necessity of searching alternative methods for finding explicit expressions for double transforms of the distributions in the two-sided boundary crossing problem. It is clear that we should not expect simple expressions here. The algorithm proposed in the present article for finding the functions $Q_{i}, i=0,1,2$, differs from methods of the previous publications by the fact that it does not use the Wiener-Hopf factorization. At the same time, we consider a rather broad class of random walks with the jump distribution belonging to the class $\mathcal{R}$ introduced in [1]. This class is characterized by the following condition: At least one of the functions $\mathbb{E}\left(\exp \left\{\lambda X_{1}\right\} ; X_{1}<0\right)$ or $\mathbb{E}\left(\exp \left\{\lambda X_{1}\right\} ; X_{1}>0\right)$ is rational. The class $\mathcal{R}$ is dense in the sense of weak convergence in the set of all distribution functions. It is easy to see that if, for instance, the function $\mathbb{E}\left(\exp \left\{\lambda X_{1}\right\} ; X_{1}<0\right)$ is rational then, for $y<0$, we have

$$
\mathbb{P}\left(X_{1}<y\right)=\sum_{k=1}^{m} M_{k}(y) e^{\alpha_{k} y}, \quad \operatorname{Re} \alpha_{k}>0,
$$

where $M_{k}(y)$ are some polynomials. The expressions of the form (4) are conventionally called exponential polynomials.

It is also known that, for the distributions of the class $\mathcal{R}$, the factorization components are explicitly expressible in terms of zeros and poles of the function $1-z \varphi(\lambda)$, but we do not use this fact.

The method proposed below for obtaining double transforms uses some tricks from [6].

\section{FORMULAS FOR DOUBLE TRANSFORMS}

Assume that

$$
\mathbb{E}\left(\exp \left\{\lambda X_{1}\right\} ; X_{1}<0\right)=\frac{R_{m}(\lambda)}{P_{k}(\lambda)},
$$

where $R_{m}$ and $P_{k}$ are polynomials of degrees $m$ and $k$, respectively. Here we have $m<k$ by necessity since $\mathbb{E}\left(\exp \left\{\lambda X_{1}\right\} ; X_{1}<0\right) \rightarrow 0$ as $\lambda=\operatorname{Re} \lambda \rightarrow \infty$.

Lemma. Let condition (5) be satisfied. Then, for $|z|<1$, the equation $1-z \varphi(\lambda)=0$ has exactly $k$ roots (taking their multiplicity into account) on the half-plane $\operatorname{Re} \lambda<0$.

Proof. The function $\mathbb{E}\left(\exp \left\{\lambda X_{1}\right\} ; X_{1}<0\right)$ exists and is bounded for $\operatorname{Re} \lambda \geq 0$. Therefore, all zeros of the polynomial $P_{k}(\lambda)$ lie on the half-plane $\operatorname{Re} \lambda<0$. Hence, the function

$$
r(z, \lambda)=1-\frac{z R_{m}(\lambda)}{P_{k}(\lambda)}-z \mathbb{E}\left(\exp \left\{\lambda X_{1}\right\} ; X_{1} \geq 0\right)
$$

is analytic on the left half-plane everywhere except for $k$ poles (with their multiplicity taken into account), where $k$ is a finite number. Take a rectangular contour with vertices at the points $(-C,-i C),(-C, i C)$, $(0, i C)$, and $(0,-i C)$. All zeros of the polynomial $P_{k}$ for $C$ large enough are inside this contour. If $\operatorname{Re} \lambda=0$ then $|\varphi(\lambda)| \leq 1$. We now show that this estimate is valid on the other parts of the contour considering $\varphi(\lambda)$ as a result of its analytic continuation to the left half-plane. Indeed, we have $|\lambda| \geq C$ on the other parts of the contour. Hence,

$$
\frac{R_{m}(\lambda)}{P_{k}(\lambda)} \rightarrow 0
$$

as $C \rightarrow \infty$. Recalling that

$$
\left|\mathbb{E}\left(\exp \left\{\lambda X_{1}\right\} ; X_{1} \geq 0\right)\right|<1
$$

for $\operatorname{Re} \lambda<0$, we conclude that

$$
|\varphi(\lambda)| \leq\left|\frac{R_{m}(\lambda)}{P_{k}(\lambda)}\right|+\left|\mathbb{E}\left(\exp \left\{\lambda X_{1}\right\} ; X_{1} \geq 0\right)\right| \leq 1
$$

on all parts of the contour for sufficiently large $C$. This means that, for $|z|<1$, the argument of $r(z, \lambda)=1-z \varphi(\lambda)$ has no increment after path-tracing. In view of the well-known argument principle for analytic functions, we infer that $r(z, \lambda)$ has exactly $k$ roots (taking their multiplicity into account) on the left half-plane. 
Denote by $\lambda_{1}(z), \ldots, \lambda_{k}(z)$ the zeros of the function $r(z, \lambda)$ lying on the left half-plane (the zeros in this sequence can be repeated and the number of repetitions of zero is equal to its multiplicity). Let

$$
\Lambda(z, \lambda)=\prod_{i=1}^{k}\left(\lambda-\lambda_{i}(z)\right)
$$

We need the following functions:

$$
\begin{array}{ll}
\omega_{t}^{-}(z, \lambda)=\sum_{n=0}^{\infty} z^{n} \int_{-\infty}^{t} e^{\lambda x} \mathbb{P}\left(S_{n} \in d x\right), & |z|<1, \operatorname{Re} \lambda \geq 0, \\
\omega_{t}^{+}(z, \lambda)=\sum_{n=0}^{\infty} z^{n} \int_{t}^{+\infty} e^{\lambda x} \mathbb{P}\left(S_{n} \in d x\right), & |z|<1, \operatorname{Re} \lambda \leq 0 .
\end{array}
$$

It is easy to see that, for every $t$, the equality $r^{-1}(z, \lambda)=\omega_{t}^{-}(z, \lambda)+\omega_{t}^{+}(z, \lambda)$ is valid for $|z|<1$ and $\operatorname{Re} \lambda=0$. For any $t$, the function $\omega_{t}^{-}(z, \lambda)$ is analytic on the half-plane $\operatorname{Re} \lambda>0$ and continuous on the boundary of this set, and the function $\omega_{t}^{+}(z, \lambda)$ possesses similar properties on the left half-plane.

Now we can formulate the main result of the article.

Theorem. Let condition (5) be satisfied. Then the following representations take place for $|z|<1$ :

$$
\begin{gathered}
Q_{0}(z, \lambda)=\frac{C(z, \lambda) e^{\lambda b}}{P_{k}(\lambda) \Lambda(z, \lambda)}+\omega_{b}^{-}(z, \lambda)-\frac{B(z, \lambda) e^{-\lambda a}}{P_{k}(\lambda)} \omega_{b+a}^{-}(z, \lambda), \\
Q_{1}(z, \lambda)=\frac{B(z, \lambda) e^{-\lambda a}}{P_{k}(\lambda)}, \quad \operatorname{Re} \lambda \geq 0, \\
Q_{2}(z, \lambda)=\left[\omega_{b}^{+}(z, \lambda)-\frac{C(z, \lambda) e^{\lambda b}}{P_{k}(\lambda) \Lambda(z, \lambda)}-\frac{B(z, \lambda) e^{-\lambda a}}{P_{k}(\lambda)} \omega_{b+a}^{+}(z, \lambda)\right] r(z, \lambda), \\
\operatorname{Re} \lambda \leq 0,
\end{gathered}
$$

where $B(z, \lambda)$ and $C(z, \lambda)$ are polynomials in $\lambda$ of degrees $k-1$ and $2 k-1$. The coefficients of these polynomials are the solution to the system of linear equations (12)-(14) below.

Proof. Given $|z|<1$, we introduce the function

$$
\Phi_{1}(z, \lambda)= \begin{cases}e^{\lambda a} Q_{1}(z, \lambda) P_{k}(\lambda), & \operatorname{Re} \lambda \geq 0 \\ e^{\lambda a}\left(1-Q_{0}(z, \lambda) r(z, \lambda)-Q_{2}(z, \lambda)\right) P_{k}(\lambda), & \operatorname{Re} \lambda \leq 0\end{cases}
$$

By (1), the function $\Phi_{1}(z, \lambda)$ is well defined for $\operatorname{Re} \lambda=0$. Show that this function is entire in $\lambda$ and has polynomial growth as $|\lambda| \rightarrow \infty$. This is so since the functions $P_{k}(\lambda)$ and $Q_{1}(z, \lambda)$ have no singularities if $\operatorname{Re} \lambda \geq 0$, and $P_{k}(\lambda), P_{k}(\lambda) r(z, \lambda), e^{\lambda a} Q_{0}(z, \lambda)$, and $Q_{2}(z, \lambda)$ have no singularities if $\operatorname{Re} \lambda \leq 0$.

If $\operatorname{Re} \lambda \leq 0$ then the functions $e^{\lambda a} Q_{0}(z, \lambda)$ and $e^{\lambda a} Q_{2}(z, \lambda)$ are bounded and if $\operatorname{Re} \lambda \geq 0$ then the function $e^{\lambda a} Q_{1}(z, \lambda)$ is bounded. Hence, $\Phi_{1}(z, \lambda)$ increases at infinity no faster than $O\left(|\lambda|^{k}\right)$ and, therefore, is a polynomial of degree at most $k$ which is denoted by $B(z, \lambda)$.

In view of the obvious inequality

$$
\mathbb{P}\left(S_{N} \in A, N=n\right) \leq \mathbb{P}\left(S_{n} \in A\right), A \subset(-\infty,-a),
$$

and absolute continuity of the measure $\mathbb{P}\left(S_{n} \in A\right)$ on the half-plane $(-\infty, 0)$, we infer that the density

$$
\frac{d \mathbb{P}\left(S_{N}<x, N=n\right)}{d x}=f_{n}(x), \quad x<0,
$$


exists. Therefore,

$$
\lim _{\lambda \rightarrow+\infty} \frac{B(z, \lambda)}{P_{k}(\lambda)}=\lim _{\lambda \rightarrow+\infty} Q_{1}(z, \lambda) e^{\lambda a}=\lim _{\lambda \rightarrow+\infty} \int_{-\infty}^{-a} e^{\lambda(x+a)} \sum_{n=1}^{\infty} z^{n} f_{n}(x) d x=0
$$

by the Riemann-Lebesgue lemma. This means that $B(z, \lambda)$ is a polynomial of degree at most $k-1$. Thus,

$$
Q_{1}(z, \lambda)=\frac{B(z, \lambda) e^{-\lambda a}}{P_{k}(\lambda)}
$$

Hence, relation (10) is established.

We now introduce the function

$$
\Phi_{2}(z, \lambda)=\left\{\begin{array}{lr}
\left(\omega_{b}^{+}(z, \lambda)-\frac{Q_{2}(z, \lambda)}{r(z, \lambda)}-\frac{B(z, \lambda) e^{-\lambda a}}{P_{k}(\lambda)} \omega_{b+a}^{+}(z, \lambda)\right) P_{k}(\lambda) \Lambda(z, \lambda) e^{-\lambda b} \\
\left(Q_{0}(z, \lambda)-\omega_{b}^{-}(z, \lambda)+\frac{B(z, \lambda) e^{-\lambda a}}{P_{k}(\lambda)} \omega_{b+a}^{-}(z, \lambda)\right) P_{k}(\lambda) \Lambda(z, \lambda) e^{-\lambda b} \\
& \text { if } \operatorname{Re} \lambda \geq 0 .
\end{array}\right.
$$

This function is well defined on the imaginary axis since (1) implies the relation

$$
\begin{aligned}
& \omega_{b}^{+}(z, \lambda)+\omega_{b}^{-}(z, \lambda)-\frac{Q_{2}(z, \lambda)}{r(z, \lambda)} \\
& \quad=Q_{0}(z, \lambda)+Q_{1}(z, \lambda)\left(\omega_{b+a}^{+}(z, \lambda)+\omega_{b+a}^{-}(z, \lambda)\right) \\
& \quad=Q_{0}(z, \lambda)+\frac{B(z, \lambda) e^{-\lambda a}}{P_{k}(\lambda)}\left(\omega_{b+a}^{+}(z, \lambda)+\omega_{b+a}^{-}(z, \lambda)\right) .
\end{aligned}
$$

As in consideration of $\Phi_{1}(z, \lambda)$, we show that the function $\Phi_{2}(z, \lambda)$ is entire and has polynomial growth as $|\lambda| \rightarrow \infty$. First we show that $\Phi_{2}(z, \lambda)$ is an entire function. This is actually so since the functions

$$
e^{-\lambda b} \omega_{b}^{+}(z, \lambda), \frac{e^{-\lambda b} Q_{2}(z, \lambda) P_{k}(\lambda) \Lambda(z, \lambda)}{r(z, \lambda)}, B(z, \lambda), \text { and } e^{-\lambda(b+a)} \omega_{b+a}^{+}(z, \lambda)
$$

have no singularities if $\operatorname{Re} \lambda \leq 0$, and the functions

$$
e^{-\lambda b} Q_{0}(z, \lambda), \omega_{b}^{-}(z, \lambda) e^{-\lambda b}, \text { and } \omega_{b+a}^{-}(z, \lambda) e^{-\lambda(b+a)}
$$

have no singularities if $\operatorname{Re} \lambda \geq 0$. It is easy to see that $\Phi_{2}(z, \lambda)$ increases at infinity no faster than $O\left(|\lambda|^{2 k}\right)$, i.e., $\Phi_{2}(z, \lambda)$ is a polynomial which is denoted by $C(z, \lambda)$.

Thus, for $\operatorname{Re} \lambda \geq 0$, we have

$$
C(z, \lambda)=\left(Q_{0}(z, \lambda)-\omega_{b}^{-}(z, \lambda)+\frac{B(z, \lambda) e^{-\lambda a}}{P_{k}(\lambda)} \omega_{b+a}^{-}(z, \lambda)\right) P_{k}(\lambda) \Lambda(z, \lambda) e^{-\lambda b}
$$

and, for $\operatorname{Re} \lambda \leq 0$,

$$
C(z, \lambda)=\left(\omega_{b}^{+}(z, \lambda)-\frac{Q_{2}(z, \lambda)}{r(z, \lambda)}-\frac{B(z, \lambda) e^{-\lambda a}}{P_{k}(\lambda)} \omega_{b+a}^{+}(z, \lambda)\right) P_{k}(\lambda) \Lambda(z, \lambda) e^{-\lambda b},
$$

which yields both representations (9) and (11).

Since the relations

$$
e^{-\lambda b} Q_{0}(z, \lambda) \rightarrow 0, \omega_{b}^{-}(z, \lambda) e^{-\lambda b} \rightarrow 0, \frac{B(z, \lambda)}{P_{k}(\lambda)} \omega_{b+a}^{-}(z, \lambda) e^{-\lambda(b+a)} \rightarrow 0
$$


hold as $\operatorname{Re} \lambda \rightarrow+\infty$, we have $\frac{C(z, \lambda)}{P_{k}(\lambda) \Lambda(z, \lambda)} \rightarrow 0$. Hence, $C(z, \lambda)$ is a polynomial of degree at most $2 k-1$.

We are left with finding exactly $3 k$ coefficients of the polynomials $B(z, \lambda)$ and $C(\lambda, z)$.

First, suppose that all roots of the polynomial $\Lambda(z, \lambda)$ are prime. Observe that all the quantities $Q_{0}\left(z, \lambda_{i}(z)\right), \omega_{b}^{+}\left(z, \lambda_{i}(z)\right)$, and $\omega_{b+a}^{+}\left(z, \lambda_{i}(z)\right)$ are finite. From (10) and (1) it follows that

$$
\frac{B\left(z, \lambda_{i}(z)\right) e^{-\lambda_{i}(z) a}}{P_{k}\left(\lambda_{i}(z)\right)}=Q_{1}\left(z, \lambda_{i}(z)\right)=1-Q_{2}\left(z, \lambda_{i}(z)\right) .
$$

Inserting $\lambda=\lambda_{i}(z)$ into (11) implies

$$
1-Q_{2}\left(z, \lambda_{i}(z)\right)=1+\frac{C\left(z, \lambda_{i}(z)\right) e^{\lambda_{i}(z) b} S_{i}(z)}{P_{k}\left(\lambda_{i}(z)\right)},
$$

where

$$
S_{i}(z)=\left[\frac{r(z, \lambda)}{\Lambda(z, \lambda)}\right]_{\lambda=\lambda_{i}(z)}
$$

So we have $k$ equations

$$
B\left(z, \lambda_{i}(z)\right) e^{-\lambda_{i}(z) a}-C\left(z, \lambda_{i}(z)\right) e^{\lambda_{i}(z) b} S_{i}(z)=P_{k}\left(\lambda_{i}(z)\right), \quad i=1, \ldots, k .
$$

Let

$$
P_{k}(\lambda)=\prod_{i=1}^{k}\left(\lambda-p_{i}\right),
$$

where all roots $p_{1}, \ldots, p_{k}$ are prime. Multiply both sides of (11) by $e^{-\lambda b}$ and put $\lambda=p_{i}$. Note that the quantities $Q_{2}\left(z, p_{i}\right) e^{-p_{i} b}$ are finite for all $i$. The same is true for the right-hand side of (11). Obviously, $r\left(z, p_{i}\right)=\infty$; therefore,

$$
\begin{aligned}
& {\left[C(z, \lambda)+B(z, \lambda) e^{-(b+a) \lambda} \omega_{b+a}^{+}(z, \lambda) \Lambda(z, \lambda)\right]_{\lambda=p_{i}}=0, i=1, \ldots, k,} \\
& {\left[e^{-b \lambda} \omega_{b}^{+}(z, \lambda)-\frac{\lambda-p_{i}}{P_{k}(\lambda)} \frac{d}{d \lambda}\left(\frac{C(z, \lambda)}{\Lambda(z, \lambda)}\right.\right.} \\
& \left.\left.+B(z, \lambda) e^{-(b+a) \lambda} \omega_{b+a}^{+}(z, \lambda)\right)\right]_{\lambda=p_{i}}=0, \quad i=1, \ldots, k .
\end{aligned}
$$

We thus obtain $3 k$ equations (12)-(14) to define $3 k$ unknown coefficients of the polynomials. It is easy to see that the coefficients of these equations are analytic functions in the variable $z$ in the disk $|z|<1$. The same holds for the determinant of this system. Hence, this determinant vanishes only at finitely many points $z$.

In the case of the multiple roots $\lambda_{i}(z)$ and $p_{j}$, we need obviously to differentiate the corresponding equations before substitution. Observe also that, like $\varphi(\lambda)$, the function $\varphi^{\prime}(\lambda)$ is analytic on the left half-plane everywhere except for finitely many poles. Therefore, $\varphi^{\prime}(\lambda)$ can vanish on this half-plane only at some finite set of points $\lambda_{1}, \ldots, \lambda_{T}$. Hence, among the roots $\lambda_{i}(z)$, there may be multiple only for the points $z_{i}=\left[\varphi\left(\lambda_{i}\right)\right]^{-1}, i=1, \ldots, T$. Clearly, for these $z$, we can define the coefficients of the polynomial $B(z, \lambda)$ by continuity.

As an example, we calculate the functions $Q_{i}$ in the case when $X_{1}$ has the density

$$
f(x)=\frac{\alpha}{2} e^{-\alpha|x|}
$$


Then

$$
\varphi(\lambda)=\frac{\alpha^{2}}{\alpha^{2}-\lambda^{2}}, \quad r(z, \lambda)=\frac{\lambda^{2}+(z-1) \alpha^{2}}{\lambda^{2}-\alpha^{2}} .
$$

Here

$$
\begin{gathered}
k=1, \quad p_{1}=-\alpha, \quad \lambda_{1}(z)=-\alpha \sqrt{1-z}, \\
S_{1}(z)=\frac{2 \lambda_{1}(z)}{\lambda_{1}(z)^{2}-\alpha^{2}} .
\end{gathered}
$$

Put $B(z, \lambda)=b_{0}(z)$ and $C(z, \lambda)=c_{1}(z) \lambda+c_{0}(z)$. Denote

$$
\begin{aligned}
& W_{0}(z)=\omega_{b}^{+}\left(z, p_{1}\right) \\
& W_{1}(z)=e^{-(b+a) p_{1}} \omega_{b+a}^{+}\left(z, p_{1}\right), \\
& W_{2}(z)=e^{-(b+a) p_{1}}\left[\frac{d \omega_{b+a}^{+}(z, \lambda)}{d \lambda}\right]_{\lambda=p_{1}} .
\end{aligned}
$$

Then the system of equations (12)-(14) takes the form

$$
\left\{\begin{array}{l}
\frac{b_{0}(z) e^{-\lambda_{1}(z)(b+a)}}{S_{1}(z)}-c_{1}(z) \lambda_{1}(z)-c_{0}(z)=\frac{\left(\lambda_{1}(z)+\alpha\right) e^{-\lambda_{1}(z) b}}{S_{1}(z)} \\
W_{1}(z)\left(p_{1}-\lambda_{1}(z)\right) b_{0}(z)+c_{1}(z) p_{1}+c_{0}(z)=0 \\
\left((b+a) W_{1}(z)-W_{2}(z)\right) b_{0}(z)+\frac{c_{1}(z) p_{1}+c_{0}(z)}{\left(p_{1}-\lambda_{1}(z)\right)^{2}}=-e^{-b p_{1}} W_{0}(z) .
\end{array}\right.
$$

From the second and third equations it follows that

$$
b_{0}(z)=\frac{e^{-b p_{1}} W_{0}(z)\left(p_{1}-\lambda_{1}(z)\right)}{W_{1}(z)+\left(W_{2}(z)-(b+a) W_{1}(z)\right)\left(p_{1}-\lambda_{1}(z)\right)} .
$$

Summing the first and second equations, we find

$$
c_{1}(z)=\frac{\left(\lambda_{1}(z)+\alpha\right) e^{-\lambda_{1}(z) b}}{\left(p_{1}-\lambda_{1}(z)\right) S_{1}(z)}-\left(W_{1}(z)+\frac{e^{-\lambda_{1}(z)(b+a)}}{\left(p_{1}-\lambda_{1}(z)\right) S_{1}(z)}\right) b_{0}(z) .
$$

Finally, the second equation yields

$$
c_{0}(z)=W_{1}(z)\left(\lambda_{1}(z)-p_{1}\right) b_{0}(z)-p_{1} c_{1}(z) .
$$

\section{ACKNOWLEDGMENTS}

The authors express their sincere appreciation to A. A. Mogulskiı and I. S. Borisov for their useful remarks that made it possible to improve presentation. Partially supported by the Russian Foundation for Basic Research (grant 11-01-00285). 


\section{REFERENCES}

1. A. A. Borovkov, "The factorization identities and distribution properties of the supremum of sequential sums," Teor. Veroyatnost. i Primenen. 15 (3), 377-418 (1970) [Theory Probab. Appl. 15 (3), 359-402 (1970)].

2. V. I. Lotov, "Asymptotic analysis of distributions in problems with two boundaries. I," Teor. Veroyatnost. i Primenen. 24 (3), 475-485 (1979) [Theory Probab. Appl. 24 (3), 480-491 (1979)]

3. V. I. Lotov, "The asymptotic behavior of distributions connected with the exit of a nondiscrete random walk from an interval," in Limit Theorems of Probability Theory and Related Questions, Trudy Inst. Mat. 1 (Novosibirsk, Nauka, 1982), pp. 18-25 [in Advances in Probability Theory: Limit Theorems and Related Problems, (N.Y., Optimization Software, 1984), pp. 29-41].

4. V. I. Lotov and V. R. Hodzibaev, "On the probability of ruin," Izv. Akad. Nauk UzSSR, Ser. Fiz.-Mat. Nauk 3, 28-34 (1980) [in Russian].

5. W. Feller, An Introduction to Probability Theory and Its Applications. Vol. 1., (M.: Mir, 1984) [in Russian].

6. D. J. Emery, "Exit problem for a spectrally positive process," Adv. Appl. Probab. 5, 498-520 (1973).

7. J. H. B. Kemperman, "A Wiener-Hopf type method for a general random walk with a two-sided boundary," Ann. Math. Statist. 34 (4), 1169-1193 (1963). 